\title{
The importance of the dental team in palliative care
}

\section{Opinion}

For those patients with dementia, palliative care means a group of essential actions involving different professionals and their specializations, independent of the phase the patient is in. The disease may be life threatening or not, however it is progressive, debilitating and incurable. It is of upmost importance that the patient and family are provided with clear information about the concepts and objectives of the palliative treatment. The family together with the patient must be covered in all their needs with the proper orientation. ${ }^{1,2}$

The population of senior citizens is growing worldwide and the oldest group is the one that is growing proportionally faster. As the population ages so does the number of senior citizenss who present chronical diseases. ${ }^{3}$ An important group within these diseases is those formed with dementia syndromes, one of the geriatric giants, since aging is the main risk factor. The dementias together with neurodegenerative diseases form the group that generates more functional disability, which causes dependency to perform basic daily activities. There are several types of dementia, and the most wellknown and feared is Alzheimer s disease. ${ }^{4}$

With the advancement of the disease, the patient presents an increase of dependence for basic daily activities increasing beyond the commitment of his autonomy. This gradual loss of functional performance can generate consequences, such as falls, malnutrition, infections from different origins, including the oral cavity, since oral hygiene is mostly affected. ${ }^{4,5}$ Advanced care planning and anticipatory treatment planning may be prudent when it is known that cognitive impairment may progress over time.

The oral cavity is vitally important to well-being, as it is directly related to nutritional health issues; preservation of the ability to feed orally is one of the goals to be achieved in palliative care, as even patients receiving nutritional support via a gastrostomy tube can benefit from comfort feeding, which is the possibility of still experiencing the pleasure of savoring one's favorite foods. ${ }^{6}$ Verbal communication is another important item to preserve and oral health contributes significantly, since speech to be well understood involves oral structures such as tongue and teeth. As long as this ability is functioning the patient is able to interact concerning adjustments, sensibility and fluency, chewing capacity, preferences and even esthetical issues. $^{7}$

It is essential that members of the Palliative Care team carry out active screening to enable early detection of signs and symptoms. The oral cavity may be a valuable clinical finding at times the only one to aid investigation of broader problems. In many cases, changes in the mouth, such as signs of dehydration, serve to screen for systemic diseases. $^{8}$

In advanced stages of dementia, characterized by the Functional Assessment Staging (FAST) 7f, patients present decreased level of consciousness. ${ }^{9}$ Symptoms like oral pain and dry mouth may be neglected, either because of their inability to report the symptom or because the oral cavity was not examined by the Palliative Care team dentist. There is a widespread culture of neglect toward thorough

\author{
Volume 3 Issue 3 - 2019
}

\author{
Oliva $A,{ }^{\prime}$ Fonseca $A^{2}$ \\ 'Dentist, Gerontology and Geriatric Brazilian Society, Rio de \\ Janeiro, Brazil \\ ${ }^{2}$ Geriatric Doctor, Rio de Janeiro, Brazil \\ Correspondence: Fonseca A, Geriatric Doctor, Gerontology \\ and Geriatric Brazilian Society, Rio de Janeiro, Brazil, \\ Email anelise1976@gmail.com
}

Received: March 28, 2019 | Published: May 13, 2019

investigation of the oral cavity during physical examination. In this context, it is worth stressing that the dentist is the provider best trained to detect abnormal findings in the oral cavity, which may cause major pain issues. ${ }^{10}$

With the presence of the dentist, after the diagnosis, all the complex interventions permitted at that time should be performed. Surgical interventions are an important part of palliative dentistry. Invasive treatment like tooth extraction is not commonly considered palliative treatment, but essentially it is, because when correctly indicated the surgery is mandatory for the patient's comfort and well-being. The multiple extractions of compromised teeth in advanced stages of dementia may avoid focal infections, allows better maintenance of the oral hygiene and hydration and also promotes easier oral feeding. This is the concept of functional edentulism.

Keep in mind, with the passage of time, only simple interventions will be possible. The goal is the maintenance of the quality of oral care, which includes oral hygiene and hydration. This is matter of dignity until to life's end. Patients with very advanced disease, in which dental treatment might lead to greater clinical instability, should be treated less invasively. Individualized treatment planning avoids complex techniques and prioritizes simpler interventions.

In short, palliative care in dentistry has a wide scope of practice. In addition to controlling oral symptoms, dentists can direct therapy as appropriate to prioritize pain control, proper hydration, and preservation of oral feeding for those with serious incurable diseases, as well as for patients with reduced autonomy or high dependence as a result of prolonged illness, as in some neurodegenerative diseases.

\section{Acknowledgements}

None.

\section{Conflicts of interest}

The authors declare that they have no conflict of interest and both of them had the same participation on paper.

\section{References}

1. Palliative care definition.

2. Palliative care. Noncommunicable diseases and their risk factors. 
3. Demographic ageing.

4. Carlsson CM, Gleason CE, Puglielli L, et al. Dementia. Chapter 65 Dementia Including Alzheimer's disease. Hazzard's Geriatric Medicine and Gerontology.

5. Wiseman MA. Palliative care dentistry. Gerodontology. 2000;17(1):49-51.

6. Geddis-Regan A, Walton G. A guide to treatment planning in complex older adults. Br Dent J. 2018;225(5):395-399.

7. Ferrucci L, Fabbri E. Inflammageing: chronic inflammation in ageing, cardiovascular disease and frailty. Nat Rev Cardiol. 2018;15(9):505-522.
8. Mulk BS, Chintamaneni RL, Mpv P, et al. Palliative dental care - a boon for debilitating. J Clin Diagn Res. 2014;8(6):ZE01-ZE06.

9. Auer S, Reisberg B. The GDS/FAST staging system. Int Psychogeriatr. 1997;9 Suppl 1:167-171.

10. Delwel S, Binnekade TT, Perez RSGM, et al. Oral hygiene and oral health in older people with dementia: a comprehensive review with focus on oral soft tissues. Clin Oral Investig. 2018;22(1):93-108. 